\title{
The Convergence and Optimization Performance of the Simplified Artificial Fish School Algorithm
}

\author{
Fuqing Zhao ${ }^{1, a}$, Guoqiang Yang ${ }^{1, b}$, Yi Zhang, ${ }^{2, c}$, Weimin $\mathrm{Ma}^{3, \mathrm{~d}}$, Chuck Zhang ${ }^{4, \mathrm{e}}$ and Houbin \\ Song ${ }^{1, f}$ \\ ${ }^{1}$ School of Computer and Communication Technology, Lanzhou University of Technology, Lanzhou 730050, \\ China \\ ${ }^{2}$ School of Mechanical Engineering, Xijin University, Xi'an 710123, China \\ ${ }^{3}$ School of Economics and Management, Tongji University, Shanghai 200092, China \\ ${ }^{4} \mathrm{H}$. Milton Stewart School of Industrial \& Systems Engineering, Georgia Institute of Technology, Atlanta, GA \\ 30332, USA \\ afzhao2000@hotmail.com, ${ }^{\mathrm{b} e s t i v a l i n p @ 163 . c o m, ~}{ }^{\mathrm{c}} 1049440546 @ q q . c o m,{ }^{\mathrm{d}}$ mawm@tongji.edu.cn, ${ }^{\mathrm{e}} \mathrm{chuck} . z h \mathrm{a}$ \\ ng@isye.gatech.edu, ${ }^{\mathrm{f}} 523712418 @ q q . c o m$
}

Keywords: artificial fish school algorithm; convergence; optimization; neighbor structure

\begin{abstract}
As a new evolution optimization, artificial fish school algorithm has the features of high convergence speed and good performance on solving combinatorial optimization. To improve the optimization performance of the artificial fish school algorithm, a simplified artificial fish school algorithm is proposed. In the forage process, artificial fish can directly move to the optimal position in its vision distance to fasten the search speed. In the clustering behaviors, the center of the neighbor domain can be replaced by the center of the whole fish school. In the clustering process, the optimal position is replaced by optimal position of the fish school. Therefore, the computing process can proceed only by the distance of the center and optimal position of the fish school. The distance of the neighbor distance and current fish school, the utmost value of the neighbor and the distance of optimal artificial fish and all the fish neighbor domain can be simplified to shorten the running time. Simulation results show that the SAFSA has good performance on convergence speed, running time and optimization ability to high dimension function.
\end{abstract}

\section{Introduction}

Optimization algorithms have been widely applied to solve a lot of numerical optimization problems which originating from the real world problems. For the real-parameter numerical global optimization problems, heuristic and metaheuristic algorithms have already expressed excellent search performance [1]. And most of them are widely used in the real-life engineering optimization [2-4]. Classical meta-heuristics are described as follows, such as genetic algorithm [5], simulated annealing [6], differential evolution [7,8], particle swarm optimization [9], artificial bee colony [10], and other typical hybrid evolution computation algorithm.

Artificial fish school algorithm (AFSA) also is an evolutionary computational model which is based on swarm intelligence. AFSA is developed by [11] who has been inspired by the research of the artificial livings. Similar to PSO [9], AFSA is also an optimizer based on population. The system is initialized firstly in a set of randomly generated potential solutions, and then performs the search for the optimum one iteratively. Whereas the AFSA does not possess the crossover and mutation processes used in GAs [12], it finds the optimum solution by swarms following the best fishes [13, 14]. Compared to GAs, the AFSA has much more profound intelligent background and could be performed more easily. Based on its advantages, the AFSA is not only suitable for science research, but also engineering applications, in the fields of evolutionary computing, optimization and many others [15-19]. In recent years there have been a lot of reported works focused on the AFSA which has been applied widely in the function optimization [18], artificial neural network training [20], 
fuzzy control [21] and some other fields. Some improved AFSA algorithms have also been developed. Similar hybrid AFSA algorithms are proposed by introducing new operations into AFSA systems. After a hybrid probability is assigned to each fish, the algorithm selects a certain number of fishes into a pool according to the hybrid probabilities at each stage of iteration. The fishes in the pool are randomly separated into school. Each school reproduces two children by operation mechanism for the fish school. Then the children are used to replace their parents of the previous fishes to keep the number of schools unchanged.

Certain amount of researches and practitioners have devoted in the domain of parameters, neighbor structure and behavior adjustment of artificial fish school algorithm to propose the novel improved artificial fish school algorithm. Literature [13] adopted adjustable parameters to enhance the lower searching precision and convergence speed in later evolution process for basic AFSA. Literature [22] proposed an improved AFSA to enable the algorithm having high stability and global performance. From the literatures on AFSA, we can see that most of the research focusing on the application of AFSA to certain problem. The basic theory of the AFSA need put more energy on it. The research on the algorithm itself and optimization still be in experiment process. Meanwhile, for AFSA, it has the shortcoming of the weak balance of exploitation and exploration ability, of the randomly searching ability in the lateness evolution process, of the lower searching precision, to affect the quality and efficiency.

In this paper, an improved mechanism for basic AFSA has been implemented to simplify the mechanism of the fish school in searching process, and to improve the searching precision and convergence speed. It can be proved by the experiments that the algorithm improves the efficiency of tradition AFSA algorithm and enhance the success rate to seek the optimal solution. The paper is organized as follows. Section 2 provides the mechanism of the SAFSA. In section 3, Convergence analysis of the SAFSA is proposed in searching process Simulation result is given in section 4 . Finally, Section 5 concludes the paper.

\section{A Simplified Artificial Fish School Optimization Algorithm (SAFSA)}

\subsection{The Mechanism of the SAFSA}

In artificial fish school algorithm, the model of the artificial can be depicted by the following class.

Class Artificial_fish \{

Various:

float AF_X[n]; $\quad / / A F$ 's position

float AF_step; $\quad / /$ the distance that AF can moue for each step

float AF_visual; $\quad$ //the visual diatance of $A F$

float try_number; $\quad$ //attempt time in the behavior of prey

float AF_delta; Ithe condition of jamming

Functions:

float AF_foodconsistence( ); //the food consistence of AF's current position

float AF_move( ); $\quad$ //AF move to the next position

float AF_follow( ); $\quad$ //the behavior of follow

float AF_prey( ); $\quad$ //the behavior of prey

float AF_swarm( ); $\quad$ //the behavior of swarm

float AF_evaluate( ); $\quad$ /levaluate and select the behavior

float AF_init( ); $\quad$ //to initialize the AF

Aritificial_fish( );

Virtual Aritificial_fish( );

\} ;

By the class description, the information and the behaviors are encapsulated in one certain class and its behaviors can be observed by the partners. In the forage process, artificial fish can directly move to the optimal position in its vision distance to fasten the search speed. In the clustering behaviors, the center of the neighbor domain can be replaced by the center of the whole fish school. In 
the clustering process, the optimal position was replaced by optimal position of the fish school. Hence, the computing process can proceed only by the distance of the center and optimal position of the school. The distance of the neighbor distance and current fish school, the utmost value of the neighbor and the distance of optimal artificial fish and all the fish of neighbor domain can be simplified to shorten the running time.

With the above melioration, the basic artificial fish can be improved as:

$$
\begin{aligned}
& X_{i}=X_{p}+\text { Step } \times \frac{X_{c}-X_{i}}{\left\|X_{c}-X_{i}\right\|}+\text { Step } \times \frac{X_{\text {max }}-X_{i}}{\left\|X_{\text {max }}-X_{i}\right\|} \\
& \left\{\begin{array}{l}
\text { Visual }=\text { Cisual } \times a+\text { Visual }_{\text {min }} \\
\text { Step }=\text { Step } \times a+\text { Step }_{\text {min }} \\
a=\exp \left[-30 \times\left(t / T_{\text {max }}\right)^{s}\right]
\end{array}\right.
\end{aligned}
$$

where,

$X_{c}$ — the center of the whole artificial fish,

$X_{\max }$ - the optimal position of the whole artificial fish finding in current iteration,

$X_{p}$ - the position of the artificial fish after forage operation,

$t$ - current iteration number,

$T_{\max }$ — the maximum iteration mumber,

$X_{\max }$ - the maximum value of the searching range,

$s$ - an integer greater than $1, s \in[1,30]$.

In Eq. 1, the first component is the result of foraging operation of artificial fish. It is represents that the artificial fish have confirmation and confidence to the forage operation of the school. The second component is the clustering behaviors which represents that the artificial fish moves forward along the center. The third part is the chasing behaviors which represents that the fish move toward the global optimal position. The equation combines the forage behaviors, clustering behaviors and chasing behavior to adjust the position and status of the next step according to the result of forage, the center of the school and the optimal position of the school to fasten the searching speed and shorten the running time. Meanwhile, the random move behaviors were included in the forage behaviors to guarantee the ability of the escaping the local optimum in the running process.

In general situation, the initial value of the Visual was set as $X_{\max } / 4$, Step as Visaul/8, Visual $_{\min }=0.001$ Step $_{\min }=0.0002$. The visual and the step consist of three components. The Visual and the Step kept as the maximum value at the beginning of the running of the algorithm. The value diminished gradually till minimum. The algorithm balanced global searching ability and local searching ability effectively with the high convergence speed and accuracy simultaneously.

\subsection{Description of the SAFSA Algorithm}

Step 1. Initialize the population of the artificial fish $M$, visual and the step, crowded degree $\delta$, maximum number of try iteration try_number, the maximum number of iteration.

Step 2. Calculate the fitness of each candidate and compare with the value posted on announce boarder. If the value is better than that on the boarder, then replace the value previously posted on the boarder with the current calculation result.

Step 3. Calculate the visual and step with Eq. 2.

Step 4. The forage behavior for each candidate.

Step 5. The candidates update the position according to Eq. 1.

Step 6. Verify the terminated conditions (the predetermined iteration number or the fitness unchanged for predetermined iteration). If the condition was satisfied, then the optimal result, which includes the status of artificial fish and the function fitness, will be posted and the algorithm will be terminated. Otherwise, the program converted to Step 2. 


\section{Convergence Analysis of the SAFSA}

In the basic artificial fish school algorithm, the forage behavior is the fundament of the convergence of the algorithm; clustering behaviors strengthen the stability and the global performance of the algorithm; chasing behavior enhances the rapidity and global performance of the algorithm; the evaluation selection for the behavior provide the guarantee for the convergence speed and stability.

\subsection{Rudimentary knowledge}

Definition 1. $P$ is a $k \times k$ transition probability matrix $\left\{P_{i, j}, i, j=1,2, \cdots, k\right\}$. The stochastic process with one finite state space $\left\{S=s_{1}, s_{2}, \cdots, s_{k}\right\}$ is called as Markov chains which has a state of time homogeneous for transition probability matrix $P$. If $\forall n \quad i, j=1,2, \cdots, k$, $i_{0}, i_{1}, \cdots, i_{n-1} \in\{1,2, \cdots, k\}$, then:

$$
\begin{aligned}
& P\left(X_{n+1}=S_{j} \mid X_{0}=s_{i_{0}}, X_{1}=s_{i_{1}}, \cdots, X_{n-1}=s_{i_{n-1}}, X_{n}=S_{i}\right) \\
& =P\left(X_{n+1}=s_{j} \mid X_{n}=s_{i}\right) \\
& =p_{i . j}
\end{aligned}
$$

Definition 2. $\forall s_{1}, s_{2} \in S$, if

$$
P\left(X_{m+n}=s_{j} \mid X_{m}=s_{i}\right)>0
$$

then for state space $\left(S=s_{1}, s_{2}, \cdots, s_{k}\right.$ ), Markov chains which has a state of timehomogeneous with the transition probability matrix $\mathrm{P}$ is irreducible. The following equation is:

If Markov chains $\left(X_{0}, X_{1}, \cdots\right)$ is a Markov chains with a state of timehomogeneous and irreducible, then:

$$
P\left(X_{m+n}=s_{j} \mid X_{m}=s_{i}\right)=\left(P^{n}\right)_{i . j}>0
$$

Definition 3. $\forall s_{i} \in S$, if

$$
d\left(s_{i}\right)=\operatorname{gcd}\left\{n \geq 1:\left(P^{n}\right)_{i . i}>0\right\}=1,
$$

then Markov chains $\left(X_{0}, X_{1}, \cdots\right)$ is an nonperiodic Markov chains.

$\operatorname{gcd}\left\{a_{1}, a_{2}, \cdots\right\}$ is the greatest common measure for $a_{1}, a_{2}, \cdots$ the period of the states $s_{i} \in S$ is the greatest common measure for the set of the transfer number which represents the probability of the Markov chains returning to the state of $s_{i}$.

Lemma 1. Strict positive Markov chains is the irreducible and nonperiodic Markov chain

Lemma 2. Let $P^{\prime}$ is a $n$ rank reducible random matrix. In other word, by same column transfer and row transfer, $P^{\prime}=\left(\begin{array}{cc}C & 0 \\ R & T\end{array}\right)$. C is a $m$ rank primitive random matrix, $R, T \neq 0$, then

$$
P^{\prime \infty}=\lim _{k \rightarrow \infty} P^{\prime k}=\lim _{k \rightarrow \infty}\left(\begin{array}{cc}
C^{k} & 0 \\
\sum_{i=0}^{k-1} T^{i} R C^{k-i} & T^{k}
\end{array}\right)=\left(\begin{array}{ll}
C^{\infty} & 0 \\
R^{\infty} & 0
\end{array}\right)
$$

where $P^{\prime \infty}$ is a stable random matrix, and $P^{\prime \infty}=1^{\prime} p^{\prime \infty}, p^{\prime \infty}=p^{\prime 0} . P^{\prime \infty}$ is an unique and has no relations with the initiation status. $P^{\prime \infty}$ satisfies $p_{i}^{\infty}>0,1 \leq i \leq m$, and $p_{i}^{\infty}=0, m<i \leq n$. 


\subsection{Analysis for state space for SAFSA}

If representing the $x_{i}$ in $X=\left(x_{1}, x_{2}, \cdots, x_{n}\right)$ of artificial fish with $M$ binary string, that is quantizing $\left[\underline{x}_{i}, \bar{x}_{i}\right]$ with $2^{M}$ discrete value, then the precision can be expressed as $\varepsilon=\left(\bar{x}_{i}-\underline{x}_{i}\right) / 2^{M}$. So the convergence of the artificial fish can be analyzed with real number coding. Let required precision as $\varepsilon$, the searching space is set as discrete space with the size of $|s|=\prod_{i=1}^{n}\left(\bar{X}_{i}-\underline{X}_{i}\right) / \varepsilon$. The fitness of each candidate $X \in S$ is Fitness $(X)$. Let $F=\{\operatorname{Fitness}(X) \mid X \in S\}$, it is obviously that $|F| \leq|S|$, so $Y$ can be expressed as $F=\left\{F_{1}, F_{2}, \cdots, F_{|F|}\right\}$, with $F_{1}>F_{2}>\cdots>F_{|F|}$. the set $S$ can be divided into certain non void subset according to the fitness of candidate, where

$$
\begin{aligned}
& S_{i}=\left\{X \mid X \in S \text { and Fitness }(X)=F_{i}\right\} \\
& \sum_{i=1}^{|F|}\left|S_{i}\right|=|S| ; S_{i} \neq \varnothing, \forall i \in\{1,2, \cdots,|F|\} ; S_{i} \cap S_{j}=\varnothing, \forall i \neq j ; \bigcup_{i=1}^{|F|} S_{i}=S
\end{aligned}
$$

To each random $X_{i} \in S_{i}, X_{j} \in S_{j}$, it is certain that

Fitness $\left(X_{i}\right)>$ Fitness $\left(X_{j}\right), \quad i<j$

Fitness $\left(X_{i}\right)=$ Fitness $\left(X_{j}\right), \quad i=j$

Fitness $\left(X_{i}\right)<$ Fitness $\left(X_{j}\right), \quad i>j$

It is obviously that $F_{1}$ is the global optimal solution $F^{*}$, and all the candidate with fitness $F^{*}$ are included in subset $S_{1}$.

In the evolution process of artificial fish algorithm, the number of artificial fish $p=\left\{X_{1}, X_{2}, \cdots, X_{N}\right\}$ in the school is stable and unchanged. Let $P$ as the aggregation of all the school, because it is allowable that the candidates are same in the school, so the number of school could be

$$
|P|=\left(\begin{array}{c}
|S|+N-1 \\
N
\end{array}\right)
$$

To measure the performance of the school, define the food density of the position of the school as

$$
\operatorname{Fitness}(p)=\max \left\{f\left(X_{i}\right) \mid i=1,2, \cdots, N\right\}
$$

Then $F_{|F|} \leq \operatorname{Fitness}(p) \leq F_{1}, \forall p \in P$, so we can divide set $P$ as non void subset $\left\{P_{i}\right\}$,

$$
\begin{aligned}
& P_{i}=\left\{p \mid p \in P \text { and Fitness }(p)=F_{i}\right\}, i=1,2, \cdots,|F| \\
& \sum_{i=1}^{|F|}\left|P_{i}\right|=|P| ; P_{i} \neq \varnothing, \forall i \in\{1,2, \cdots,|F|\} ; P_{i} \cap P_{j}=\varnothing, \forall i \neq j ; \bigcup_{i=1}^{|F|} P_{i}=P
\end{aligned}
$$

where $P_{1}$ includes all the school with fitness $F^{*}$.

Let $P_{i j}$ as the $j$ th school in $P_{i}, i=1,2, \cdots,|F|, j=1,2, \cdots,\left|P_{i}\right|$. in the process of the evolution, the transfer from $P_{i j}$ to $P_{k l}$ can be express as $P_{i j} \rightarrow P_{k l}$. Let $p_{i j . k l}$ as the transfer probability from $P_{i j}$ to $P_{k l}$, $p_{i j . k}$ as the transfer probability from $P_{i j}$ to random school of $P_{k}, p_{i . k}$ as the random school of $P_{i}$ to random school of $P_{k}$, it is obviously that

$$
p_{i j . k}=\sum_{l=1}^{\left|P_{k}\right|} p_{i j . k l}, \sum_{k=1}^{|F|} p_{i j . k}=1, p_{i . k} \geq p_{i j . k}
$$




\subsection{Proof of the convergence feature of SAFSA}

Definition 4. An evolution algorithm converges to global optimum, iff

$\lim _{t \rightarrow \infty} \operatorname{Pr}\left\{\right.$ Fitness $\left.\left(P^{t}\right)=F^{*}\right\}=1$

where, $\operatorname{Pr}$ is the probability, $P^{t}$ is the th population.

Theorem 1. The searching process of artificial fish school algorithm is a timehomogeneous Markov chain.

Proof. According to the running mechanism of artificial fish school algorithm, its searching domain is located in the population space $S$ with limited space. And in the searching process, the emergent of the next generation $p^{t+1}$ depends only on current population, and have no connections with previous generation $\left(p_{t-1}, p_{t-2}, \cdots, p_{1}, p_{0}\right)$. Hence, the condition probability from one certain population $p^{t}$ to another population $p_{t+1}$ will not be affected by the initiation change. Form the definition of Markov chain, the searching process has the feature of Markov property, in other words, it has no afterward effects and satisfies the criterion of Markov property. So the searching process of the artificial fish school optimization can be depicted by timehomogeneous Markov chain with finite state.

Theorem2. In the artificial fish school algorithm, $\forall i, k \in\{1,2, \cdots,|F|\}$,

$$
\begin{cases}p_{i . k}>0 & k \leq i \\ p_{i . k}=0 & k>i\end{cases}
$$

Proof. $\forall p_{i j} \in P_{i}, i=1,2, \cdots,|F|, j=1,2, \cdots,\left|P_{i}\right|, \exists X^{*}=\left(x_{1}^{*}, x_{2}^{*}, \cdots, x_{n}^{*}\right) \in P_{i j}$, Fitness $\left(X^{*}\right)=F_{i}$

$\forall p_{k l} \in P_{k}, k=1,2, \cdots,|F|, l=1,2, \cdots,\left|P_{k}\right|, \exists X^{\prime}=\left(x_{1}^{\prime}, x_{2}^{\prime}, \cdots, x_{n}^{\prime}\right) \in P_{k l}$, Fitness $\left(X^{\prime}\right)=F_{k}$

It is supposed that under the evolution mechanism, $P_{i j}$ transfer to $P_{k l}$. If $P_{i j}$ is the thenerations, then $P_{k l}$ is the $(t+l)$-th generation, marked them with $P^{t}$ and $P^{t+1}$ respectively.

In the basic artificial fish school algorithm, the elite strategy was adopted by posting the optimal status of the candidate on the broad. According to the above definition and hypothesis, the optimal artificial fish of $P^{t}$ is $X^{*}$, which was saved on Bulletin_Board, that is Bulletin_Board $=X^{*}$, the form of population $P^{t}$ will not be altered. For $P^{t}$, after forage, clustering, chasing and random behavior, the next generation $P^{t+1}$ can be evolved. Compare optimal artificial fish $X^{\prime}$ with the value of Bulletin_Board, if the fitness of $X^{\prime}$ is better than Bulletin_Board, then Bulletin_Board $=X$; otherwise keep the value on the Bulletin_Board. Put the Bulletin_Board on the utmost left of the population, however, the Bulletin_Board will not participant in the evolution process, so

$$
\begin{aligned}
& \text { Fitness }\left(\text { Bulletin }_{\text {Board }}\right) \geq \text { Fitness }\left(X^{*}\right) \\
& \Rightarrow \text { Fitness }\left(P^{t+1}\right) \geq \text { Fitness }\left(P^{t}\right) \\
& \Rightarrow k \leq i \\
& \Rightarrow \forall k>i, p_{i j . k l}=0 \\
& \Rightarrow \forall k>i, p_{i j . k}=\sum_{l=1}^{\left|P_{k}\right|} p_{i j, k l}=0 \\
& \Rightarrow \forall k>i, p_{i . k}=0 .
\end{aligned}
$$

In each iterations of the artificial fish school algorithm, it is by forage, clustering, chasing and random behavior to find optimum. The principal of the behavior can select according to the fastest evolution speed and keep the progress value. If the behavior is selected as chasing, the clustering behavior will be selected next until no progress has been made on the chasing behavior; the forage 
behavior will be selected if the previous two behaviors have no progress. The random behavior will be exerted if no progress has been made on the chasing, clustering and forage. The situation can be analyzed into three cases.

(1) Let the probability of chasing behavior is $p_{\text {fallow }} \geq 0$. if $X^{*}$ select the chasing behavior, then $p_{\text {fallow }}>0$, and if the position of the optimal neighbor have high food density and uncrowned, $X^{*}$ will move towards the position of the optimal neighbor which have high food density in the latter position $X^{\prime}$. And if $\forall k<i, p_{i . k}>0$, then Fitness $\left(X^{\prime}\right) \geq \operatorname{Fitness}\left(X^{*}\right)$.

(2) Let the probability of clustering behavior $p_{\text {school }} \geq 0$. If $X^{*}$ select the clustering behavior, then $p_{\text {school }}>0$, and if the food density of the neighbor center is not crowded, $X^{*}$ will move towards center of neighbor. The food density of the position which was moved is higher than that of moved before, then $\forall k<i, p_{i, k}>0$, Fitness $\left(X^{\prime}\right) \geq \operatorname{Fitness}\left(X^{*}\right)$.

(3) Let the probability of the forage is $p_{\text {prey }}=1-p_{\text {school }}-p_{\text {fallow }}$. The artificial fish $X^{*}$ explore the random selection status in its searching space. There are two situations: (1) the food density of the position $X^{\prime}$ which is the status of selected is higher than that of $X^{*}$, and the probability of the cases is $p_{p p y}$, if $\forall k<i, p_{i . k}>0$, then Fitness $\left(X^{\prime}\right) \geq \operatorname{Fitness}\left(X^{*}\right)$. (2) the food density of the position $X^{\prime}$ which is the status of selected is lower than that of $X^{*}$, and the probability of the case is $p_{p n}=1-p_{p p y}$, then the candidate will re-select the position and will try certain numbers try_number with the probability of $\left(p^{p n}\right)^{\text {try_number }}$. If the conditions still can not be satisfied, the candidate will select the random behavior. Let the probability of random move is prandom, the probability of the food density for moved afterward higher than move before is $p_{\text {better }}=(1 / 2) \cdot\left(1-\left(p^{p n}\right)^{\text {try number }} \times p_{\text {random }}\right) \geq 0$; and if $p_{\text {better }} \geq 0$, the proof is finished; if $p_{\text {better }}=0$, it is mean that the artificial fish reach the local optimum. By controlling the number of try_number , the artificial fish school can keep the diversity of the school, guarantee the population move randomly, and escape the local optimum.

Form the basic artificial fish school algorithm, it is concluded that the total probability of selecting one of the behaviors is 1 , that is $p_{\text {prey }}+p_{\text {school }}+p_{\text {fallow }}=1$, integrate the above three cases, we can see that $\forall k<i, p_{i . k}>0$, so the proposition is correct.

The theorem states that the population with lower fitness can move to the population with same fitness or even higher fitness, and can not move from population with higher fitness to lower fitness. So, once the algorithm moves into set $P_{1}$, it can not move out of the evolution process.

Theorem3. The basic artificial fish school algorithm has global convergence property.

Proof. According to the conclusion of theorem1, each $P_{i}, i=1,2, \cdots,|F|$ can be view as one certain status of finite Markov chains. According to the conclusion of theorem 2, the transfer matrix of the Markov chains can be expressed as

$$
P^{\prime}=\left(\begin{array}{cccc}
p_{1.1} & p_{1.2} & \cdots & 0 \\
p_{2.1} & p_{2.2} & \cdots & 0 \\
\vdots & \vdots & \ddots & \vdots \\
p_{|F| .1} & p_{|F| .2} & \cdots & p_{|F| \cdot|F|}
\end{array}\right)=\left(\begin{array}{cc}
C & 0 \\
R & T
\end{array}\right),
$$

where, $R=\left(p_{2.1} p_{3.1} \ldots p_{|F| \cdot \mid}\right)^{T}>0, T \neq 0, C=\left(p_{1.1}\right)=(1) \neq 0$.

According to Lemma 2, 


$$
P^{\prime \infty}=\lim _{k \rightarrow \infty} P^{\prime k}=\lim _{k \rightarrow \infty}\left(\begin{array}{cc}
C^{k} & 0 \\
\sum_{i=0}^{k-1} T^{i} R C^{k-i} & T^{k}
\end{array}\right)=\left(\begin{array}{cc}
C^{\infty} & 0 \\
R^{\infty} & 0
\end{array}\right),
$$

where, $C^{\infty}=1, R^{\infty}=(11 \ldots 1)^{T}$. So, $P^{\prime \infty}$ is a stable random matrix, and $P^{\prime^{\infty}}=\left(\begin{array}{cccc}1 & 0 & \cdots & 0 \\ 1 & 0 & \cdots & 0 \\ \vdots & \vdots & \ddots & \vdots \\ 1 & 0 & \cdots & 0\end{array}\right)$.

From the proof process, we can see that the probability of the candidate stay on non global optimum is approaching to 0 . In other words, when the evolution time goes to $\infty$, the algorithm will converge to optimum with probability of 1 .

According to Definition 4, $\lim _{t \rightarrow \infty} \operatorname{Pr}\left\{\right.$ Fitness $\left.\left(P^{t}\right)=F^{*}\right\}=1$. The proposition is proofed.

\section{Simulation Results and Analysis}

\subsection{Set up the experiment}

To testify the performance of improved artificial fish school algorithm, the following 5 benchmark function was used as the test cases. The simulation program tools is $\mathrm{C}++, \mathrm{CPU}$ of computer is P4 (1.7GHz).

$$
\begin{aligned}
& f_{1}(x)=\sum x_{i}^{2} \\
& f_{2}(x)=\frac{1}{4000} \sum_{i=1}^{n} x_{i}^{2}-\prod_{i=1}^{n} \cos \left(\frac{x_{i}}{\sqrt{i}}\right)+1 \\
& f_{3}(x)=\sum_{i=1}^{n-1}\left[100\left(x_{i+1}^{2}-x_{i}\right)^{2}+\left(1-x_{i}\right)^{2}\right] \\
& f_{4}(x)=\sum_{i=1}^{n}\left[x_{i}^{2}-10 \cos \left(2 \pi x_{i}\right)+10\right] \\
& f_{5}(x)=\sum_{i=1}^{n}\left(\sum_{j=1}^{i} x_{i}^{2}\right)
\end{aligned}
$$

Table 1. Parameters of test functions

\begin{tabular}{lllll}
\hline Function & Dimension & Searching range & $\begin{array}{l}\text { Optimum } \\
\text { value }\end{array}$ & precision \\
\hline$f 1$ & 10 & {$[-100,100]$} & 0 & $10^{-5}$ \\
$f 2$ & 10 & {$[-600,600]$} & 0 & $10^{-2}$ \\
$f 3$ & 10 & {$[-100,100]$} & 0 & 10 \\
$f 4$ & 10 & {$[-100,100]$} & 0 & 10 \\
$f 5$ & 10 & {$[-100,100]$} & 0 & $10^{-5}$ \\
\hline
\end{tabular}

The parameters of the algorithm in the test cases are as follows:

population $=15$, try_number $=5, \delta=11$, for $f_{1}, f_{3}$, and $f_{5}$, Visual $=25$, Step $=3$, for $f_{2}$, Visual $=150$, Step $=18$, for $f_{4}$, Visual $=2.5$, Step $=0.3$. In SAFSA, Visual ${ }_{\min }=0.001$, Step $p_{\text {min }}=0.0002, s=3$, Visual and Step were adjusted according to Eq. 2. The maximum iteration is 1000. the parameters and objective precision were set as Table 1. The test cases used AFSA and SAFSA as the computing tools to calculate the minimum objective, the test results was the average fitness by 50 independent running. 
Performance evaluation was adopted the following methods. (1) Evaluate the convergence speed and precision under the constant evolution iteration number. (2) Evaluate the iteration number to meet the predefined precision under the pre-stated precision.

\subsection{Simulation results and analysis}

The predefined evolution iteration number is set as 1000. The algorithm was calculated independently for 50 times. The results are shown in Table 2 and Fig. 1 to Fig. 5. The average optimization results and the best optimization obtained by SAFSA are better than that of AFSA, and have a high convergence speed and lower average running time. The average evolution curves of $f_{1}$ $f_{5}$ are shown in Fig.1 to Fig. 5. In each figure, the vertical ordinate is expressed as the general logarithm of the average function, while evolution generation is shown in horizontal ordinate. The optimization results of AFSA and SAFSA are shown as dotted line and solid line respectively.

From the figure, the convergence speed and optimization precision are accelerated after 400 generations. The convergence precision and convergence speed of SAFSA have a significant improvement than that of AFSA.

Table 2. The results of two optimization algorithm

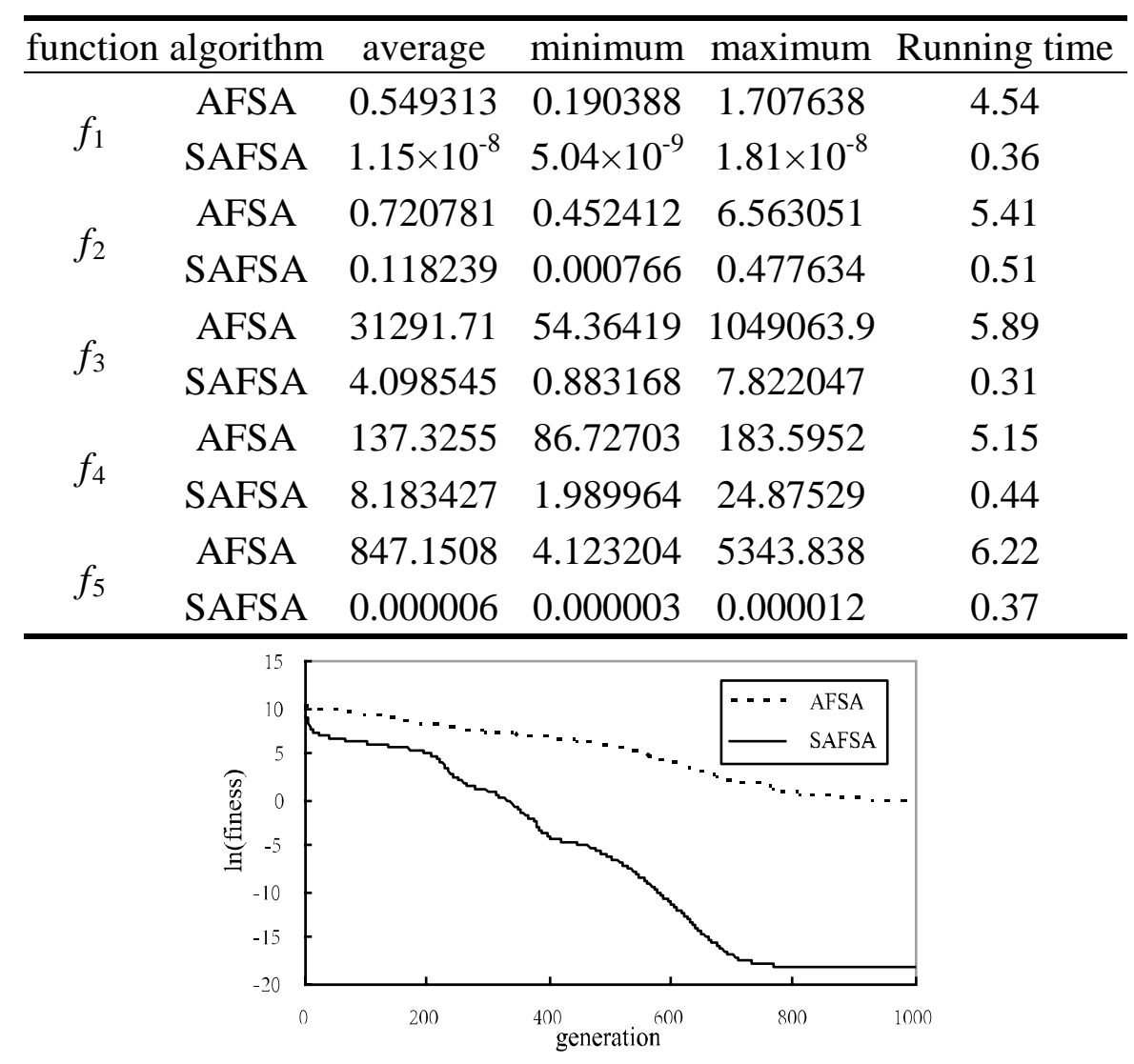

Figure 1 . The convergence curve of $f_{1}$

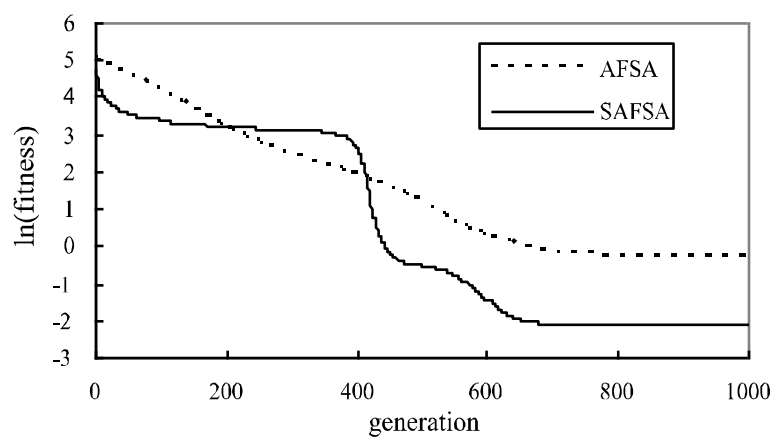

Figure 2. The convergence curve of $f_{2}$ 


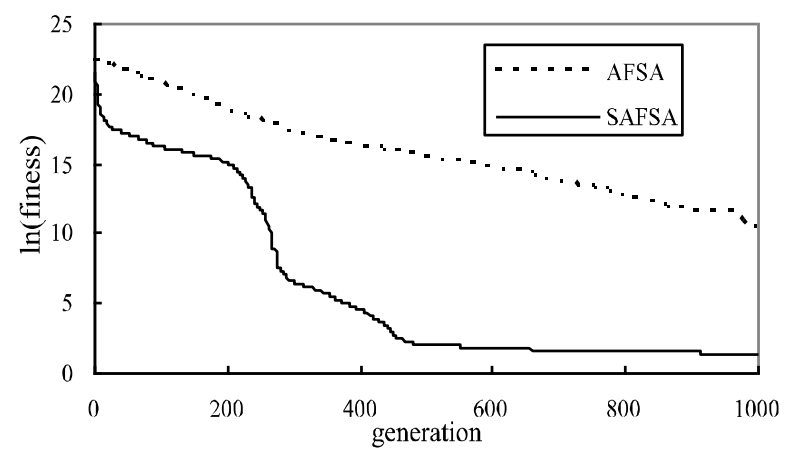

Figure 3. The convergence curve of $f_{3}$

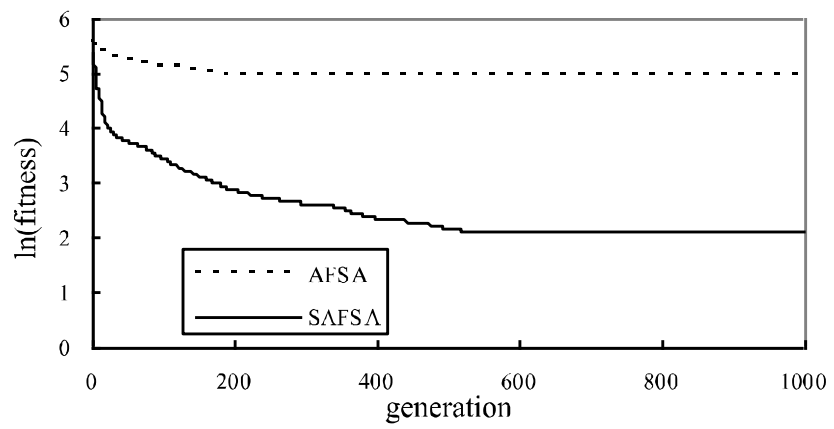

Figure 4. The convergence curve of $f_{4}$

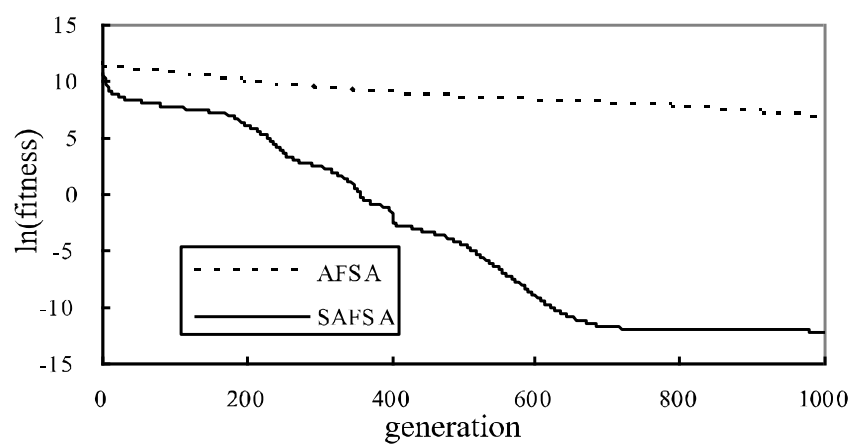

Figure 5. The convergence curve of $f_{5}$

Table 3. The evolution iteration number under predefined convergence precision

\begin{tabular}{cccccc}
\hline Function & Algorithm & Success rate $(\%)$ & $\begin{array}{c}\text { Average } \\
\text { running time }\end{array}$ & $\begin{array}{c}\text { Minimum } \\
\text { iteration }\end{array}$ & $\begin{array}{c}\text { Maximum } \\
\text { iteration }\end{array}$ \\
\hline \multirow{2}{*}{$f_{1}$} & AFSA & 0 & - & - & - \\
& SAFSA & 100 & 606 & 589 & 630 \\
$f_{2}$ & AFSA & 0 & - & - & - \\
& SAFSA & 100 & 157 & 30 & 464 \\
$f_{3}$ & AFSA & 0 & - & - & - \\
& SAFSA & 98 & 421 & 365 & 557 \\
$f_{4}$ & AFSA & 0 & - & - & - \\
& SAFSA & 82 & 267 & 76 & 560 \\
$f_{5}$ & AFSA & 0 & - & - & - \\
& SAFSA & 100 & 631 & 617 & 647 \\
\hline
\end{tabular}

The evolution iterations number under predefined convergence precision. The iteration number for the test function running independently is shown on Table 3. “-_" represents that the iteration number greater than 1000. Success rate is the running number which achieve the convergence precise divided 
by the total test time.

From Table 3, we can see that: (1) Success rate of AFSA to the five test function is zero, and AFSA have no effect to functions with high dimension. (2) The success rate of SAFSA is $100 \%$ to $f_{1}, f_{2}$ and $f_{5}$; the success rate to $f_{3}$ and $f_{4}$ are $98 \%$ and $82 \%$, respectively. (3) The average iteration number is in 650. From the experiment, we can draw the conclusion that the SAFSA has the good performance of stable convergence property.

\section{Conclusion}

SAFSA can implement three behaviors in one certain iteration on chasing forage results, the center of population position and the optimum position of the whole population to guarantee the algorithm move to the global optimum position. Furthermore, the random move behavior was incorporated into forage behavior to avoid the candidate trapping into local optimum, and strengthen the global searching ability of the algorithm. Moreover, the dynamic adjustment of the visual and step of artificial fish is introduced to balance the global exploration and local exploitation to improve the adaptability and the optimization precision. Simulation results show that the SAFSA has good performance on convergence speed, running time and optimization ability to high dimension function.

\section{Acknowledgements}

This work was financially supported by the National Natural Science Foundation of China under grant numbers 61663023. It was also supported by the Key Research Programs of Science and Technology Commission Foundation of Gansu Province (2017GS10817), Wenzhou public welfare science and technology project (G20170016), General and Special Program of the Postdoctoral Science Foundation of China, the Science Foundation for Distinguished Youth Scholars of Lanzhou University of Technology under grant numbers 2012M521802, 2013T60889, and J201405, respectively.

\section{References}

[1] Zhao F, Shao Z, Wang J, et al. A hybrid optimization algorithm based on chaotic differential evolution and estimation of distribution [J]. Computational \& Applied Mathematics, 2017, 36(1): 433-458.

[2] Shao Z, Pi D, Shao W. An extended continuous estimation of distribution algorithm for solving the permutation flow-shop scheduling problem [J]. Engineering Optimization, 2017: 1(1) 1-22.

[3] Zhao F, Liu H, Zhang Y, et al. A discrete Water Wave Optimization algorithm for no-wait flow shop scheduling problem [J]. Expert Systems with Applications, 2018, 91(1): 347-363.

[4] Zhao F, Liu Y, Zhang Y, et al. A hybrid harmony search algorithm with efficient job sequence scheme and variable neighborhood search for the permutation flow shop scheduling problems [J]. Engineering Applications of Artificial Intelligence, 2017, 65(1): 178-199.

[5] Hsieh S-T, Sun T-Y, Liu C-C. Potential offspring production strategies: An improved genetic algorithm for global numerical optimization [J]. Expert Systems with Applications, 2009, 36(8): 11088-11098.

[6] Javidrad F, Nazari M. A New Hybrid Particle Swarm and Simulated Annealing Stochastic Optimization Method [J]. Applied Soft Computing, 2017, 60(1): 634-654.

[7] Shao W, Pi D. A self-guided differential evolution with neighborhood search for permutation flow shop scheduling [J]. Expert Systems with Applications, 2016, 51(1): 161-176.

[8] Zhao F, Shao Z, Wang J, et al. A hybrid differential evolution and estimation of distribution algorithm based on neighbourhood search for job shop scheduling problems [J]. International Journal 
of Production Research, 2017, 54(4): 1-22.

[9] Zhao F, Liu Y, Zhang C, et al. A self-adaptive harmony PSO search algorithm and its performance analysis [J]. Expert Systems with Applications, 2015, 42(21): 7436-7455.

[10] Cui L, Li G, Wang X, et al. A ranking-based adaptive artificial bee colony algorithm for global numerical optimization [J]. Information Sciences, 2017, 417(1): 169-185.

[11] Li X-L. An optimizing method based on autonomous animats: fish-swarm algorithm [J]. Systems Engineering-Theory \& Practice, 2002, 22(11): 32-38.

[12] Mitchell M. An introduction to genetic algorithms [J]. An Introduction to Genetic Algorithms, 1996, 24(4-5): 325-336.

[13] Liao C, Yan Z. Optimal deployment in sensor networks based on hybrid artificial fish school algorithm [J]. Journal of Beijing University of Aeronautics \& Astronautics, 2010, 36(3): 373-377.

[14] Yu Y, Tian Y F, Yin Z F. Multiuser Detector Based on Adaptive Artificial Fish School Algorithm [J]. Journal of Electronics \& Information Technology, 2007, 2(1): 1480 - 1484.

[15] Li R Q, Wang Z Y, Xie L F, et al. Population optimization artificial fish school algorithm applied in transmission network expansion planning [J]. Power System Protection \& Control, 2010.

[16] Zhong W. Transmission Net-work Planning Based on Adaptived Artificial Fish School Algorithm [J]. Electrical Engineering, 2012.

[17] Cao J, Zhao X, Li Z, et al. Modified artificial fish school algorithm for free space optical communication with sensor-less adaptive optics system [J]. Journal of the Korean Physical Society, 2017, 71(10): 636-646.

[18] Wang L G, Shi Q H, Hong Y. Hybrid Optimization Algorithm of PSO and AFSA [J]. Computer Engineering, 2010, 36(5): 176-178.

[19] Carvalho V O. Combining K-Means and K-Harmonic with Fish School Search Algorithm for data clustering task on graphics processing units [J]. Applied Soft Computing, 2016, 41(C): 290-304. [20] Shi B, Li Y, Yu X, et al. Short-term electricity price prediction based on resilient adaptive artificial fish school algorithm and back propagation neural network hybrid algorithm [J]. Journal of Hydroelectric Engineering, 2010, 29(1): 106-113.

[21] Venayagamoorthy G K, Grant L L, Doctor S. Collective robotic search using hybrid techniques: Fuzzy logic and swarm intelligence inspired by nature [J]. Engineering Applications of Artificial Intelligence, 2009, 22(3): 431-441.

[22] Wrede F, Menezes B, Kuchen H. Fish School Search with Algorithmic Skeletons [J]. International Journal of Parallel Programming, 2018, 1(2):1-19. 